American Journal of Applied Sciences 9 (2): 208-212, 2012

ISSN 1546-9239

(C) 2012 Science Publications

\title{
Pulse-Width-Modulation DC Chopper using LabView Software
}

\author{
Mohammad K. Abuzalata \\ Division of Mechatronics, Faculty of Engineering Technology, \\ Al Balqa Applied University, Amman 11140 P.O. Box 425530, Jordan
}

\begin{abstract}
Problem statement: This study concerns with designing of Pulse Width Modulation (PWM) techniques and analyzing of their waveforms to initiate a comparative numerical study. Approach: This study enables the people interesting in developing the motional control algorithms of adjustable DC drive schemes (e.g., DC-DC converters). Results: LabView software was used as a real-talk simulation environment to design and analyze of the studied cases, such that: single pulse width modulation, multiple pulse width modulation and sinusoidal pulse width modulation. Full subVI schemes with labview had been designed to introduce for future studies. Conclusion: Analytical and numerical results have been also provided at the end of this study.
\end{abstract}

Key words: Pulse-Width-Modulation (PWM), voltage control, simulation environment, voltage source, switching-mode regulators, voltage regulation

\section{INTRODUCTION}

In many industrial applications, it is required to convert a fixed-voltage dc source into a variablevoltage dc source. A dc chopper converts directly from dc to dc and is also known as a dc-to-dc converter. A chopper can be considered as dc equivalent to an ac transformer with a continuously variable turn's ratio. Like a transformer, it can be used to step-down or stepup a dc voltage source.

Choppers are widely used for traction motor control in electric automobiles. They provide smooth acceleration control, high efficiency and fast dynamic response. Choppers can be used in regenerative braking of dc motors to return energy back into the supply and this feature results in energy savings for transportation systems with frequent stops. Choppers are used in dc voltage regulators and also used, in conjunction with an inductor, to generate a dc current source, especially for the current source inverter.

DC choppers can be used as switching-mode regulators to convert a dc voltage, normally unregulated, to a regulated dc output voltage. The regulation is normally achieved by pulse width modulation PWM at a fixed frequency and the switching device is normally a BJT, MOSFET, or IGBT the elements of switching-mode regulators (Ong, 1998).

There are various techniques to regulate dc output voltage. The most efficient method of dc voltage regulation is to incorporate Pulse-Width-Modulation (PWM) control within the choppers. The commonly used techniques are: single PWM, multiple PWM and sinusoidal PWM (Johnson and Jennings, 1994).
Concerning the programming language, Using LabVIEW, it is possible to speed up programming considerably as it is designed to take measurements, analyze data and present data to the user. LabVIEW makes it easy to maintain good architecture in the applications because encapsulation and modularity are easy to implement through the use of sub virtual instruments VIs (Ahmed, 2005).

Design of Sub VIs for PWM Control: The most efficient method of dc voltage regulation is to incorporate Pulse-Width-Modulation (PWM) control within the choppers. The commonly used techniques are.

Single PWM: In single-pulse-width modulation control, there is only one pulse per half-cycle and the width of the pulse is varied to control the chopper output voltage. Figure 1 shows the generation of gating signals and PWM control signal of dc chopper.

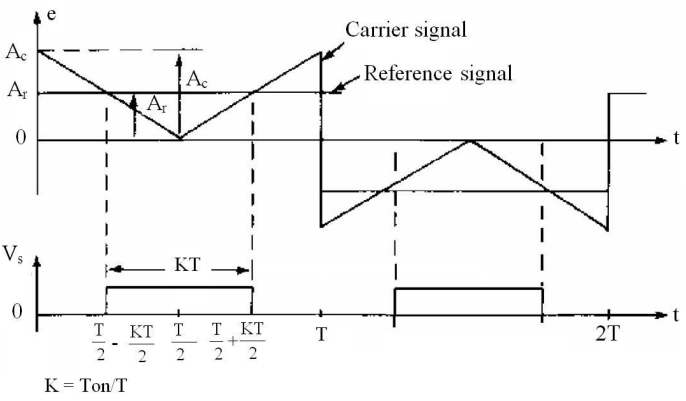

Fig. 1: Single pulse width modulation 


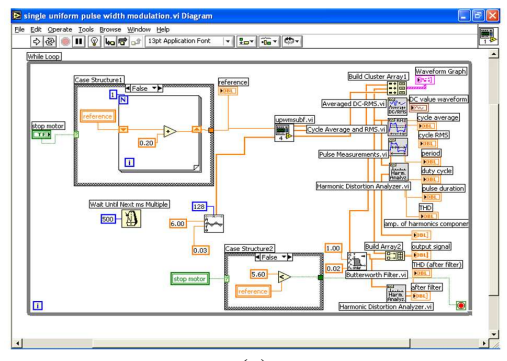

(a)

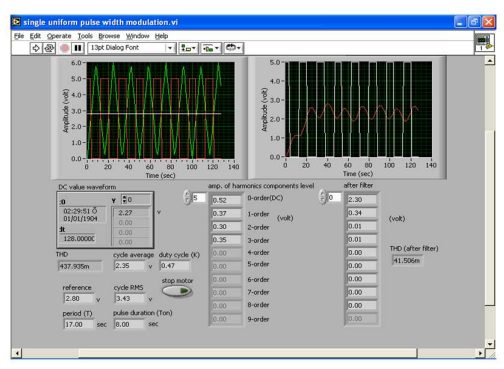

(b)

Fig. 2: The diagram and panel of single PWM

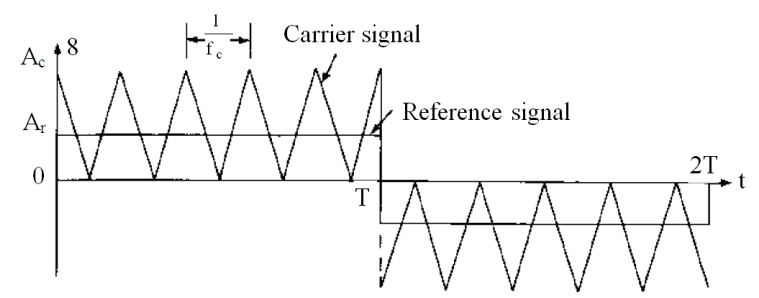

(a) Gate single generation

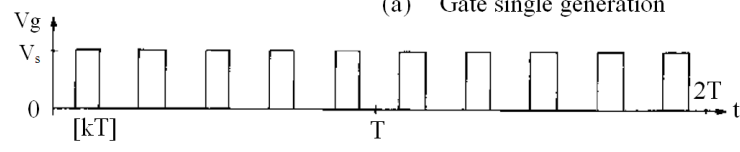

b) The control signal

Fig. 3: Multiple-pulse-width modulation

The gating signals are generated by comparing a rectangular reference signal of amplitude $A_{r}$ with a triangular carrier wave of amplitude $A_{c}$ as shown in Eq. 1. In the positive half cycle, when the reference signal amplitude is greater than the carrier signal amplitude the output voltage reach its rated value $<$ KT period $>$.

In the negative half cycle, when the carrier signal amplitude is greater than the reference signal amplitude the output voltage reach its rated value <KT period>. The frequency of the reference signal determines the fundamental frequency of output voltage. By varying $A_{r}$ from 0 to $A_{c}$, the pulse width, $K T$ (where $k=T_{o n} / T$ ), can be varied from. 0 to $T$. The ratio of $A_{r}$ to $A_{c}$ is the control variable and defined as the amplitude modulation index M. The amplitude modulation index or simply modulation index:

$$
\mathrm{M}=\mathrm{A}_{\mathrm{r}} / \mathrm{A}_{\mathrm{C}}
$$

Where:

$A_{r}=$ The amplitude of reference signal

$\mathrm{A}_{\mathrm{c}}=$ The amplitude of carrier signal

The RMS output voltage can be found from follow Eq. 2:

$$
\mathrm{Vo}=\sqrt{2 / \mathrm{T} \int_{((\mathrm{T} / 2)-\delta) / 2}^{((\mathrm{T} / 2)+\delta) / 2} \mathrm{VS}^{2} \mathrm{dt}}=\mathrm{VS} \sqrt{\delta /(\mathrm{T} / 2)}
$$

The average voltage is given by Eq. 3:

$$
\mathrm{Va}=\mathrm{Vs} * \delta /(\mathrm{T} / 2)
$$

Figure 2 below is the panel and the diagram of the single PWM virtual instrument (VI)

\section{MATERIALS AND METHODS}

Multiple PWM: The harmonic content can be reduced by using several pulses in each half-cycle of output voltage. The generation of gating signals for turning on and off of transistors is shown in Fig. 3a by comparing a reference signal with a triangular carrier wave. The control signal is shown in Fig. 3b. The frequency of reference signal sets the output frequency $f_{o}$ and the carrier frequency $f_{c}$, determines the number of pulses per halfcycle $p$, the modulation index controls the output voltage. This type of modulation is also known as uniform pulsewidth modulation UPWM. The number of pulses per halfcycle is found from Eq. 4:

$P=\frac{f_{c}}{2 f_{o}}=\frac{m_{f}}{2}$

where, $\quad m_{\mathrm{f}}=\mathrm{f}_{\mathrm{c}} / \mathrm{f}_{\mathrm{o}}$ is defined as the frequency modulation ratio.

The variation of modulation index $\mathrm{M}$ from 0 to 1 varies the pulse width, KT, from 0 to $\mathrm{T} / \mathrm{p}$ and the output voltage from 0 to $\mathrm{V}_{\mathrm{s}}$. The control signal of DC chopper is shown in Fig. 4 for UPWM.

If $\delta$ is the width of each pulse, the RMS output voltage can be found from Eq. 5:

$$
\mathrm{Vo}=\sqrt{2 \mathrm{p} / \mathrm{T} \int_{((\mathrm{T} / 2) / \mathrm{p}-\delta) / 2}^{(\mathrm{T} / 2) / \mathrm{p}+\delta) / 2} \mathrm{VS}^{2} \mathrm{dt}}=\mathrm{VS} \sqrt{\mathrm{p} \delta /(\mathrm{T} / 2)}
$$

The average voltage is Eq. 6: 
Am. J. Applied Sci., 9 (2): 208-212, 2012

$\mathrm{Va}=\mathrm{Vs} * \delta(\mathrm{T} / 2) /$

where, $\mathrm{T}$ is the periodic time of the reference signal.

Components: Four for loop-shift register, three case structures, Wait-delay, sub VI (UPWMSQU.VI), comparisons (Greater than and less than), add, divide, multiply and subtract icons, while loop, one build cluster array, [harmonic distortion analyzer][pulse measurements][averaged DC-RMS][ cycle average and RMS], $2 *$ waveform graph, one Digital Controls and eleven digital indicators, one waveform indicator, push button, one Array indicators.

Construction: The terminal of stop motor-push buttonis connected to case structure 1 and case structure2. Its local variable is connected to case structure3. Case structure1 true case include shift register (no. of iteration equal 1) its input is square amplitude local variable (decrement by 0.2 ) and its output is square amplitude terminal (which connected to square amplitude input of UPWMSQU.VI sub icon). Case structure1 false case include shift register (no. of iteration equal 1) its input is square amplitude local variable (increment by 0.2 ) and its output is square amplitude terminal. Case structure 2 true case include shift register (no. of iteration equal 1) its input is triangle frequency local variable (decrement by fo) and its output is triangle frequency terminal (which connected to triangle frequency indicator and triangle frequency input of UPWMSQU.VI sub icon).

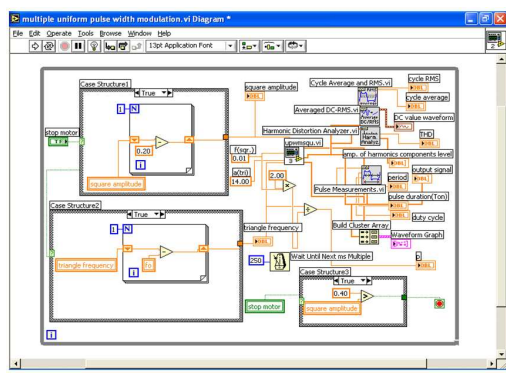

(a)

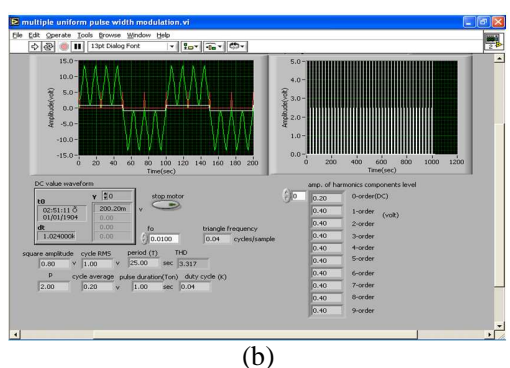

Fig. 4: The diagram (a) and (b) panel of multiple PWM VI
Case structure2 false case include shift register (no. of iteration equal 1) its input is triangle frequency local variable (increment by fo) and its output is triangle frequency terminal. Square frequency input of UPWMSQU.VI sub icon equal 0.01 and triangle amplitude equal 14 . The sub icon outputs goes to build cluster array icon (its output is waveform graph).

MPWM (Array2) output of sub icon connected to: harmonic distortion analyzer, pulse measurements, averaged DC-RMS, cycle average and RMS and output signal waveform graph.

The square frequency is multiply by 2 and the output divided to triangle frequency. The output is connected to $\mathrm{p}$ indicator. Case structure 3 true case compares square amplitude local variable with 0.4. Case structure 3 false case compares 12 with square amplitude local variable. All icons included in while loop with conditional terminal connected to the output of case structure 3 .

Operation principle: To run the circuit we must enter the value of $f_{o}$ from the panel. When we press the run button, stop motor terminal will be in false mode (activating the false case of case structure1, case structure 2 and case structure 3 ) the shift register of case structure 1 will begin with a value equal 0 and increment by 0.2 within a delay time equal $250 \mathrm{~m} \mathrm{sec}$. Also the shift register of case structure 2 will begin with a value equal 0 and increment by $f_{0}$ within a delay time equal $250 \mathrm{~m}$ sec. The variable output values of case structure 1 compared with fixed triangle wave within UPWMSQU VI sub icon (According to the fixed amplitude values of triangle wave changing the square wave amplitude values will increase the modulation index then the duty cycle $(\mathrm{K})$ will increase also the cycle average and RMS values will increase). The variable output values of case structure 2 compared with fixed square wave within UPWMSQU VI sub icon (According to the fixed frequency values of square wave changing the triangle wave frequency values will increase the frequency modulation ratio then the number of pulses per half cycle (p) will increase also the cycle average and RMS values will increase) (Ahmed, 2005). When the value of square amplitude is greater than 12, the conditional terminal of while loop will be true (the while loop stop executing).

When we press stop motor-push button- stop motor terminal will be in true mode (activating the true case of case structure 1 , case structure 2 and case structure 3 ) the shift register will begin with last value stored on it and decrement by 0.2 within a delay time equal $250 \mathrm{~m} \mathrm{sec}$. The variable output values of case structure 1 compared with fixed triangle wave within UPWMSQU.VI sub 
icon (According to the fixed amplitude values of triangle wave values changing the square wave amplitude values will decrease the modulation index then the duty cycle $(\mathrm{K})$ will decrease also the cycle average and RMS values will decrease). The variable output values of case structure2 compared with fixed square wave within UPWMSQU.VI sub icon (According to the fixed frequency values of square wave changing the triangle wave frequency values will decrease the frequency modulation ratio then the number of pulses per half cycle (p) will decrease also the cycle average and RMS values will decrease).When the value of square amplitude is less than 0.4, the conditional terminal of while loop will be true (the while loop stop executing).

Sinusoidal PWM: Instead of maintaining the width of all pulses the same as in the case of multiple-pulse modulation, the width of each pulse is varied in proportion to the amplitude of a sine wave evaluated at the center of the same pulse (Santana et al., 2002). The distortion factor and lower-order harmonics are reduced significantly. The generation of gating signals for turning on and off of transistors is shown in Fig. 5 by comparing a sinusoidal reference signal with a triangular carrier wave of frequency $f_{c}$. The control signal is shown at the same figure. This type of modulation is commonly used in industrial applications and abbreviated as SPWM. The frequency of reference signal, fr, determines the output frequency $f_{o}$ and its peak amplitude. $\mathrm{A}_{\mathrm{r}}$, controls the modulation index, $\mathrm{M}$ and then in turn the output voltage. The number of pulses per half-cycle depends on the carrier frequency.

The output voltage can be varied by varying the modulation index M. If $\delta$ is the width of $\mathrm{m}^{\text {th }}$ pulse, the RMS output voltage can be found from Eq. 7:

$$
\mathrm{Vo}=\mathrm{VS}\left[\sum_{\mathrm{M}=1}^{\mathrm{P}} \delta \mathrm{m} /(\mathrm{T} / 2)\right]^{1 / 2}
$$

The average voltage Eq. 8:

$$
\operatorname{Va}=\operatorname{VS}\left(\sum_{\mathrm{m}=1}^{\mathrm{p}} \delta \mathrm{m} /(\mathrm{T} / 2)\right)
$$

where, $\mathrm{T}$ is the periodic time of the reference signal.

Components: One for loop, comparisons (Greater or equal, greater, less than and two selects comparison), sine wave generator, triangle wave generator, case structure, two Negate icons, four Digital Controls and three Array indicators.

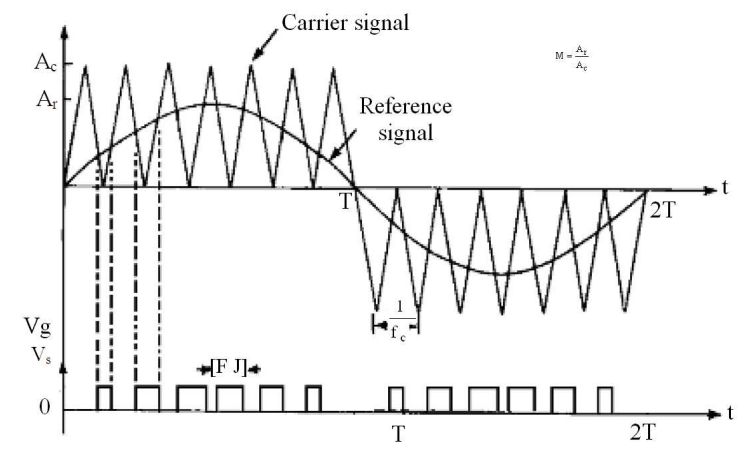

Fig. 5: Sinusoidal pulse-width modulation

Construction: Triangle wave generator output (no. of samples equal 1024, variable amplitude and frequency) is connected to: greater or equal 0 icon (its output connected to $\mathrm{s}$ input of select1 icon), $t$ input of select1 icon and negate 1 icon (its output connected to $f$ input of select1 icon). The output of select1 icon connected to the input of negate 2 icon (its output connected to $t$ input of select 2 icon) and $\mathrm{f}$ input of select2 icon. Sine wave generator output (no. of samples equal 1024, variable amplitude and frequency) is connected to the input of less than 0 icon (its output is connected to $\mathrm{s}$ input of select2 icon and to the case structure), Array2 output and to the input of case structure. The output of select2 icon connected to Array output and to the input of case structure. In case structure false case input from sine generator output and from select 2 icon are connected to greater or equal icon (its output connected to Array3). In case structure true case input from select 2 icon and from sine generator output are connected to greater or equal icon. The $t$ input of select icons is constant value equal 5 and the $\mathrm{f}$ input of select icons is constant value equal 0 . All icons are included in for loop.

Operation principle: Triangle wave will always have positive output values; because of negate1 icon operation (if the value is negative it multiplies by -1). We use negate2 and select2 icons operation for Synchronization of polarity between triangle wave and sine wave (if sine wave is positive, triangle wave will be positive and vice versa). The comparison between triangle wave and sine wave will be in-case structureoperation. If both waves are positive false case is activated (If sine wave value is greater or equal to triangle wave value, the Array 2 output will be equal to 5. If sine wave value is less than triangle wave value, the Array 2 output will be equal to 0 ). If both waves are negative true case is activated (If triangle wave value is greater or equal to sine wave value, the Array 2 output will be equal to 5 . If triangle wave value is less than sine wave value, the Array2 output will be equal to 0 ). 


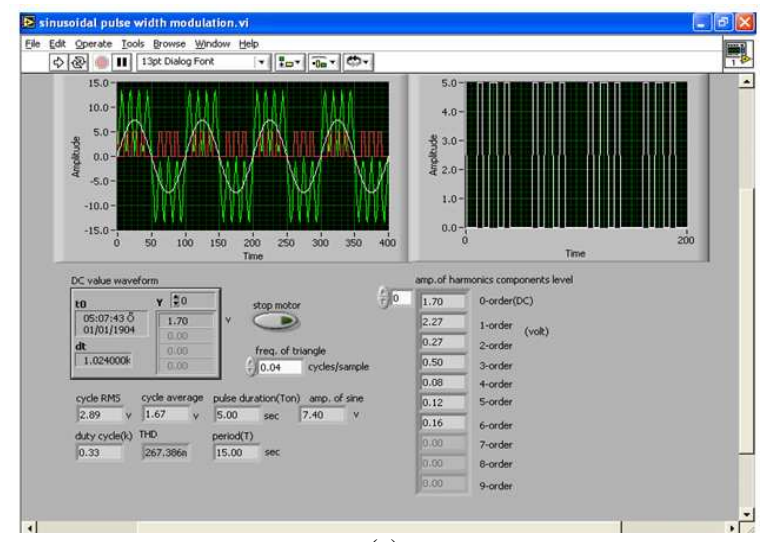

(a)

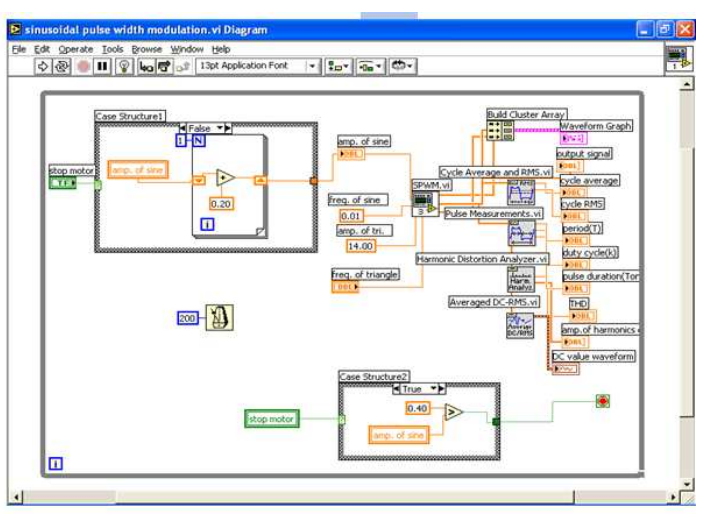

(b)

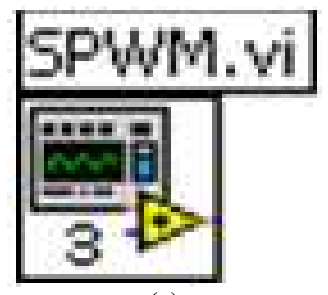

(c)

Fig. 6: The panel, diagram and sub icon of SPWM VI

Figure 6 shows the panel and the diagram and the sub icon of the SPWM VI.

\section{RESULTS AND DISCUSSION}

When taking the total harmonic distortion factor THD, the average voltage $\mathrm{V}_{\mathrm{av}}$ with respect to the modulation index $\mathrm{M}$ as one of the most important judgment criterions, a comparative study between the proposed PWM techniques in this study could be derived. The achieved analytical results have been used to plot the relations seen in Fig. 7 and 8. These figures could be obtained to verify the analytical and numerical proposed models discussed before.

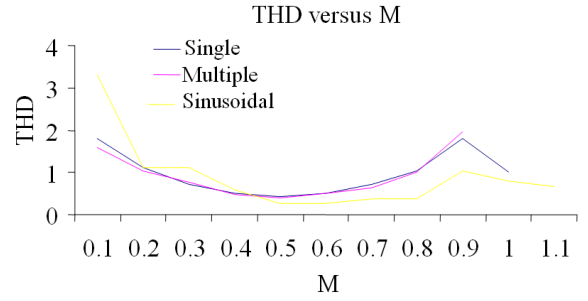

Fig. 7: THD versus $M$ (Single, multiple and sinusoidal PWM)

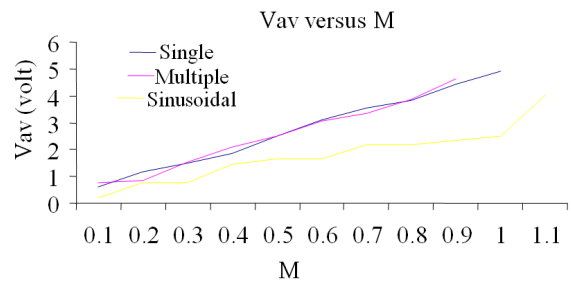

Fig. 8: $\mathrm{V}_{\mathrm{av}}$ versus $\mathrm{M}$ (Single, multiple and sinusoidal PWM)

\section{CONCLUSION}

Sinusoidal pulse width modulation achieves a low magnitude of lower order harmonics and a higher order harmonics which can be easily filtered. The highest value that can be obtained is $(0.75-0.85)$ Vs. While, the uniform pulse width modulation achieves high fundamental amplitude and very small value for the THD. The highest value that can be obtained is $0.98 \mathrm{Vs}$.

The designed sub VIs that represent the different PWM techniques have been supported by an interfacing tool to enable users modifying the control algorithm to be used in some applications.

\section{REFERENCES}

Ahmed, N.A., 2005. Modeling and simulation of ACDC buck-boost converter fed dc motor with uniform PWM technique. Elect. Power Syst. Res., 73: 363-372.

Johnson, G. and R. Jennings, 1994. LabVIEW Graphical Programming. 1st Edn., McGraw-Hill, New York, USA., ISBN: 0071501533, pp: 552.

Ong, C.M., 1998. Dynamic Simulation of Electric Machinery: using MATLAB/SIMULINK. 1st Edn., Prentice Hall PTR, New Jersey, ISBN: 0137237855, pp: 626.

Santana, J., J.L. Naredo, F. Sandoval, I. Grout and O.J. Argueta, 2002. Simulation and construction of a speed control for a DC series motor. Mechatronics, 12: $\quad 1145-1156 . \quad$ DOI: $10.1016 /$ S09574158(02)00019-3 